\title{
Self-Inflicted Oral Soft-Tissue Burn Due to Local Behavior and Treatment
}

\author{
A Dilsiz ${ }^{1}$ \\ Correspondence: \\ Alparslan DILSIZ, DDS, PhD \\ Assistant Professor, \\ Department of Periodontology, \\ Faculty of Dentistry, Atatürk University, \\ 25240-Erzurum/ TURKEY \\ E-mail:aydilsiz@yahoo.com
}

${ }^{1}$ DDS, PhD. Assistant Professor, Department of Periodontology, Faculty of Dentistry, Atatürk University, Erzurum/ TURKEY.

\begin{abstract}
Injuries to oral soft-tissues can occur due to accidental, iatrogenic, and factitious traumas. They may present as burns, ulcerations, and gingival recession. Chemical, thermal, and physical agents are the main causative agents for oral soft-tissues burns. The purpose of this case report was to illustrate the destructive nature of the local behavior and to describe the successful treatment of this case. A 20-year-old girl with severe pain and burning sensation in the left maxillary region, which was interfering with normal eating and speaking, was admitted to periodontology clinic. Upon questioning, the patient readily admitted traumatizing her gingival and cheek mucosa with alcohol. Treatment consisted of oral hygiene instruction, mechanical debridement, local antimicrobial agents, systemic analgesic and guidance for patient. The burn lesions in the oral cavity can occur at dental offices and homes. The early detection by the patient and the immediate institution of therapeutic measures ensured a rapid cure and possibly prevented further mucosal damage. In addition, we believe that guidance and education are important prophylactic tools for prevent from these local behavior.
\end{abstract}

Key words: Oral ulcer, burns, chemical burn, alcohol and gingiva. 


\section{Introduction}

The current classification of periodontal diseases includes accidental, iatrogenic, and factitious traumatic lesions (1). Although the prevalence of traumatic gingival lesions is relatively high, there are limited reports on the diagnosis and management of these injuries. Traumatic lesions, whether chemical, physical, or thermal in nature, are among the most common in the mouth. Chemical injuries of the oral soft-tissues may readily occur due to the large number of chemical substances, such as drugs and various agents, which come in contact with the oral cavity (2-12). The severity and extent of lesions caused by chemical agents depends on the concentration, type and quantity of the substance, as well as on the time of contact with the oral soft-tissues (13). Longer exposure or higher solution concentration causes epithelial necrosis and shedding (14).

Acid and alkaline substances and salts can cause considerable damage to the oral mucous membranes. They act with different pathological mechanisms to the oral softtissues (13). Acid substances cause a coagulation necrosis with the formation of a scar that limits the penetration of the acid to deep tissue layers. An initial inflammatory process, next to erythema, edema and mucosa ulceration is presented during the first $24-48$ hours after the exposure. During the two following weeks, granulation tissue is produced, that will finally develop to a cicatricial tissue during the third or fourth week. Whereas, the mechanism of action involves alkaline substances' combination with tissue protein to form proteinates, and with fats to form soap in addition to water. Thus, liquefaction necrosis occurs and reaches deeper tissue layers. Even after removing or neutralizing the etiologic substance, alkaline substances keep on destroying the tissues, resulting in ulcers and progressive lesions (15).

Only few reports in literature discussed oral soft-tissue burns from the alcohol $(7,9)$. The purpose of this case report was to illustrate the destructive nature of the local behavior and to describe the successful treatment of this case.

\section{Case report}

On March 17, 2008, a 20-year-old girl with severe pain and burning sensation in the left maxillary region, which was interfering with normal eating and speaking, was admitted to periodontology clinic.

She claimed to be in relatively good health and had not a history of drug and food allergies. She had used several brands of toothpaste during the past two years and brushed one time a day. She did not use mouthwashes and chewing gum, did not smoke and did not take alcoholic beverages.

The patient reported that she had a severe toothache of the maxillary left canine tooth the night before and called the emergency room. The emergency therapy was done and systemic analgesic medication (paracetamol $500 \mathrm{mg}$, every 6 hours) and antibiotic were prescribed. In addition, the dental history revealed that she had decided on her own to use a chemical agent as an adjunct to cease of toothache. Consequently, she used pellet of cotton wool, moistened in eau de cologne. The patient stated that she then began applying cotton pellet to the area, continuously, one after another, for about 8 hours. The intraoral examination showed an extensive white fibrin coated ulcer with surrounding erythema, from canine to first molar, involving primarily the papillary, marginal and attached gingiva with alveolar mucosal involvement (Fig.1). The lesion extended slightly lateral to the cheek mucosa. The affected mucosa was redder than usually but normal resilient and not tender. There was excessive desquamation (slough of mucosa) on the affected areas. There was little to no attachment loss in the interproximal areas of the affected teeth. Plaque control by the patient was inadequate and probing depths were within normal limits. The reason for the burn was ethyl alcohol in the eau de cologne. Dental radiographs showed that her teeth were well supported by bone; no alveolar resorption was observed, and successful endodontic treatment was done (Fig.2). On extraoral examination, her face was normal, and no palpable submandibular or cervical lymph nodes.

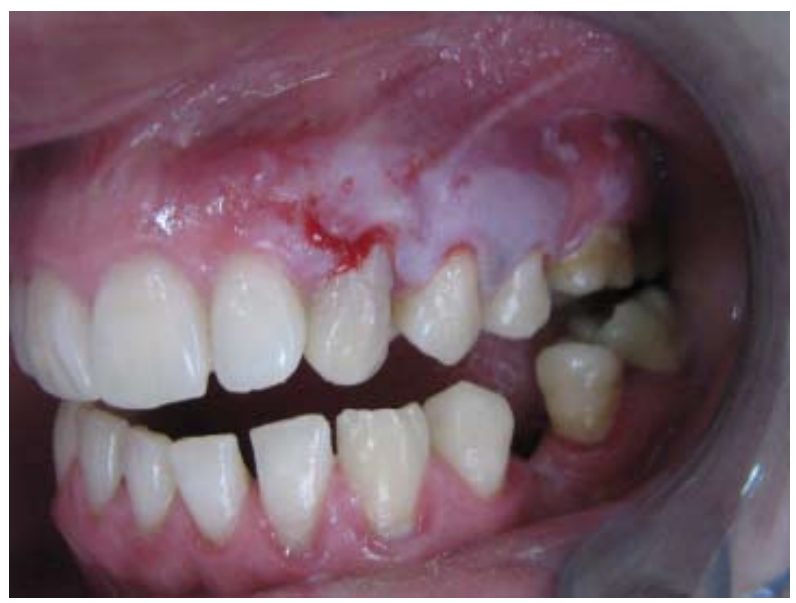

Fig. 1. Lesion on the left maxillary region caused by alcohol

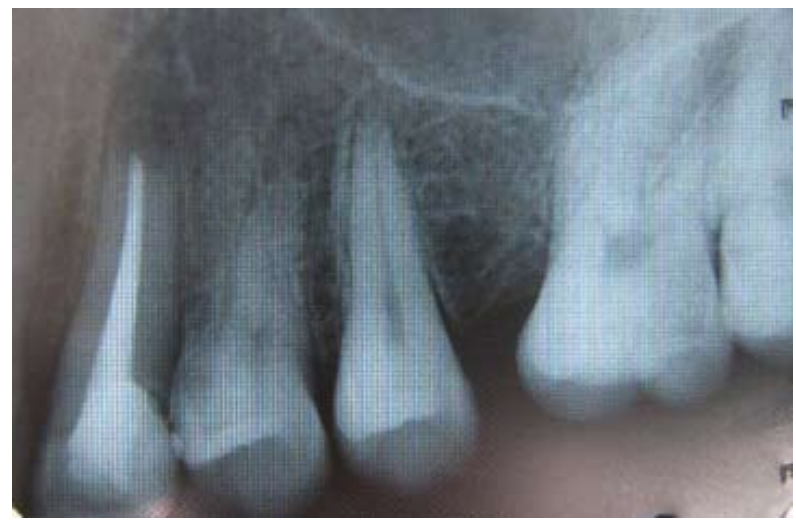

Fig. 2. Periapical radiograph 
Written informed consent was obtained from the patient after all treatment procedures had been fully explained. Treatment consisted of oral hygiene instruction, mechanical debridement, local antimicrobial agents, systemic analgesic and guidance for patient. After topical application of anesthetic spray, the area was gently cleansed and irrigated with sterilized physiological saline solution, and the patient was instructed to rinse with saline solution repeatedly, scaling and root planning was performed by quadrant. Immediately following mechanical debridement, irrigation with saline solution was repeated for to remove the tissue remnants.

Management included an analgesic (Naproxen 550mg, every 12 hours) and mouthwash (saline solution, twice daily) prescription and the application of a topical corticosteroid gel (Triamcinolone in Orabase). In addition, the patient was instructed to brush her teeth with softfilamented toothbrush twice daily, in the morning and in the evening, for 3 min each time, and bland diet was advised. Healing was uneventful after 7 days at the recall visit (Fig.3).

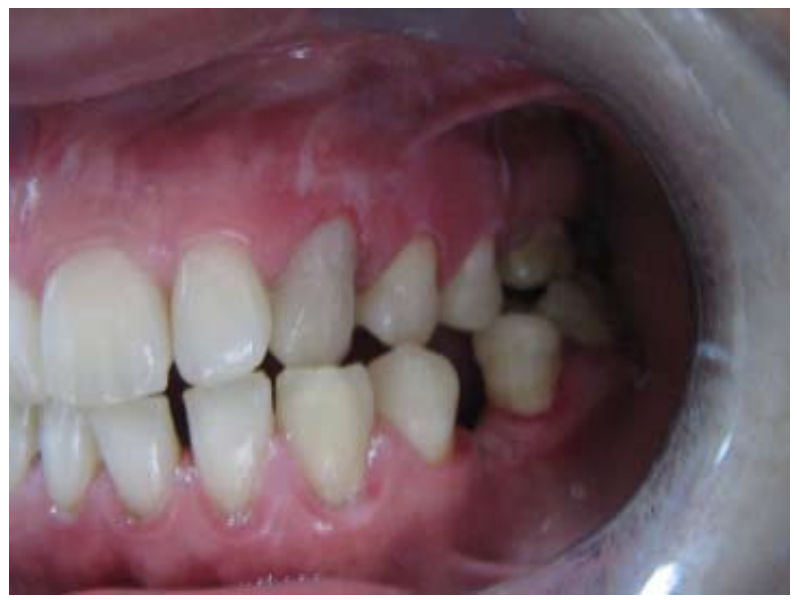

Fig. 3. View of the case 7 days after periodontal therapy

\section{Discussion}

This case reported here resulted from oral soft-tissue burn from to use a chemical agent, which was an eau de cologne, and was aggravated by the overuse. Chemical burns are often localized and are rarely confined solely to the anatomic distribution of the masticatory mucosa, as was demonstrated in this case.

In the oral cavity, chemical substances caused diffuse erosive lesions ranging from simple desquamation (slough of mucosa) to complete obliteration of the oral mucosa with extension past the basement membrane into the submucosa (5-9). In our patient, the intraoral examination revealed severe gingival and mucosal burn with diffuse sloughing of the mucosa along the whole affected area. In addition, desquamation is a common manifestation of a number of pathologic conditions such as allergic reactions, hormonal and mucocutaneous disorders.
The contact of the oral mucosa with chemical substances, usually develops during it's resolution towards the formation of inflammation (redness) and ulcerations who depending on it's localization, commit, in greater or minor measure, the phonation, mastication and swallowing $(5,6)$, as was showed in our case.

Clinical reports have singled out certain chemical substances as causes of oral mucosal burn (2-12). Dentists may cause chemical burns by careless handling of various medications, such as hydrogen peroxide $(2,5)$, sodium hypochlorite solution (4), calcium hydroxide solution (6), and formocresol solution $(3,6)$. Lesions produced vary according to the destructive properties and mode of application of the chemical agent. For example, hydrogen peroxide $(2,5)$, and calcium hydroxide solution (6) causes a localized white slough (necrotized epithelium) and erythema, while formocresol solution $(3,6)$ produces a characteristic white lesion and swelling.

The management of chemical burns requires removal of the offending agent and symptomatic therapy. Permanent removal of the agent is usually easy to accomplish in iatrogenic or accidental injury cases. In cases of selfinflicted injury it might be difficult the patient to stop the noxious behavior, as has been reported here. According to Yano et al. (15), irrigation is the emergency treatment choice to minimize the product effect and current therapy with steroids results in a very favorable prognosis. If pain is considerable, symptomatic treatment may be of benefit.

In conclusion, the present report illustrates the cologne can lead to severe focal to diffuse caustic burns of the oral mucosa as a result of the concentration of ethyl alcohol at the area. The burn lesions in the oral cavity can occur at dental offices and homes. The early detection by the patient and the immediate institution of therapeutic measures ensured a rapid cure and possibly prevented further mucosal damage. In addition, we believe that guidance and education are important prophylactic tools for prevent from these local behavior.

\section{References}

1. Armitage GC. Development of a classification system for periodontal diseases and conditions. Ann Periodontol. 1999;4:1-6.

2. Shetty K. Hydrogen peroxide burn of the oral mucosa. Ann Pharmacother. 2006;40:351.

3. Ozcelik O, Haytac MC, Akkaya M. Iatrogenic trauma to oral tissues. J Periodontol. 2005;76:1793-7.

4. Witton R, Brennan PA. Severe tissue damage and neurological deficit following extravasation of sodium hypochlorite solution during routine endodontic treatment. Br Dent J. 2005;198:749-50.

5. Rawal SY, Claman LJ, Kalmar JR, Tatakis DN. Traumatic lesions of the gingiva: a case series. J Periodontol. 2004;75:762-9.

6. Santos-Pinto L, Campos JA, Giro EM, Cordeiro R. Iatrogenic chemical burns caused by chemical agents used in dental pulp therapy. Burns. 2004;30:614-5.

7. Moghadam BK, Gier R, Thurlow T. Extensive oral mucosal ulcerations caused by misuse of a commercial mouthwash. Cutis. 1999;64:131-4.

8. Murdoch-Kinch CA, Mallatt ME, Miles DA. Oral mucosal injury 
caused by denture cleanser tablets: a case report. Oral Surg Oral Med Oral Pathol Oral Radiol Endod. 1995;80:756-8.

9. Baruchin AM, Lustig JP, Nahlieli O, Neder A. Burns of the oral mucosa. Report of 6 cases. J Craniomaxillofac Surg. 1991;19:94-6.

10. Maron FS. Mucosal burn resulting from chewable aspirin: report of case. J Am Dent Assoc. 1989;119:279-80.

11. Touyz LZ, Hille JJ. A fruit-mouthwash chemical burn. Report of a case. Oral Surg Oral Med Oral Pathol. 1984;58:290-2.

12. Rubright WC, Walker JA, Karlsson UL, Diehl DL. Oral slough caused by dentifrice detergents and aggravated by drugs with antisialic activity. J Am Dent Assoc. 1978;97:215-20.

13. Mamede RC, de Mello Filho FV. Ingestion of caustic substances and its complications. Sao Paulo Med J. 2001;119:10-5.

14. Rossi LA, Braga EC, Barruffini RC, Carvalho EC. Childhood burn injuries: circumstances of occurrences and their prevention in Ribeirao Preto, Brazil. Burns. 1998;24:416-9.

15. Yano K, Hata Y, Matsuka K, Ito O, Matsuda H. Effects of washing with a neutralizing agent on alkaline skin injuries in an experimental model. Burns. 1994;20:36-9. 